\title{
Off-Label Use in Rare Diseases: Myasthenia Gravis, Lambert-Eaton Myasthenic Syndrome and Congenital Myasthenic Syndromes
}

(@) $(\Theta \Theta$

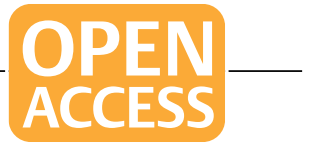

Author

Rudolf Janzen

Institut

former head of department, Neurologische Klinik am Krankenhaus Nordwest, Franfurt Main

Key words

off-label use, refractory myasthenia gravis, Lambert-Eaton myasthenic syndrome, congenital myasthenic syndrome

Bibliography

DOI https://doi.org/10.1055/s-0044-100184

Neurology International Open 2018; 2: E118-E123

(c) Georg Thieme Verlag KG Stuttgart · New York

ISSN 2511-1795

Correspondence

Prof. Dr. med. Rudolf W.C. Janzen

Landwehrweg $12 \mathrm{C}$

61350 Bad Homburg

Germany

info@rwcjanzen.de

\section{ABSTRACT}

The off-label use of licensed drugs is widespread, and the risk of adverse events is elevated, especially if the scientific evidence is low. The treatment of rare diseases $(<5 / 10,000)$ therefore may increase the risk of off-label use. The optimized standard treatment is primarily based on the regulatory decisions of the German Federal Joint Committee. The valid guidelines and the regulations of the German health system are discussed in this article. The criteria for indication and monitoring of off-label use are shown, especially focused on the problem of refractory myasthenia gravis. Escalation of treatment results regularly in off-label use. The arguments within the applicable guidelines should be followed. Some new aspects of the European regulations are likewise discussed.

\section{Background}

\section{Off-Label Use}

Off-label use occurs when a licensed drug is applied outside its intended use. The intended use of a drug is documented in the official prescribing information and is based on the related monograph of the German Federal Institute for Drugs and Medical Devices (BfArM) [1]. According to a $2017 \mathrm{EU}$ study, the general prescription of a substance for off-label use in adults is between 7-95\% in inpatient care, and between 6-75\% in the outpatient sector [2]. Numerous guidelines of the Association of the Scientific Medical Societies (AWMF) describe substances used off-label as well as the related level of evidence and assessment. Off-label use may be required for the physician if these guidelines have clearly formulated the conditions for the drug treatment, and the off-label use meets the requirements of the various opinions of the Federal Social Court or relevant opinions of the Federal Constitutional Court [3-6] or if no on-label therapy is available for the disease [7]. An assumption of costs by the German statutory health insurance (GKV) can in principle only be made if a substantiating application is made by the treating neurologist before the beginning of the therapy as well as a positive opinion of the health insurance medical service (MDK) and/or prior consent of the
GKV is available. The extent of the off-label use thus obtained in accordance within the process or outside standard procedure ("tolerated" off-label use) has not been systematically investigated in Germany. With regard to liability, it should be noted that a manufacturer who is aware of widespread off-label use of a product must be held liable in accordance with Section 84 of the German Drug Law [3].

Off-label use is generally associated with an increased risk of adverse drug reactions (ADR), as shown by the recent results of a Canadian cohort study [7]. According to the study, the average rate of ADRs in general-care on-label use patients was $12.5 / 10,000$ patient months versus $19.1 / 10,000$ patient months in a patient group with off-label use [7]. Such findings, together with the recent EU study [2], were the basis for the "Good Off-Label Use Practices" initiative of the European Brain Council [8], the core concept of which is the promotion of significantly improved pharmacovigilance, supported by the European Myasthenia Gravis Association (EuMGA).

\section{Rare diseases: myasthenia gravis}

Since 2000 , diseases with a prevalence of $<5$ out of every 10,000 people in the EU have been defined as "rare diseases". According to a statement by Orphanet regarding myasthenia gravis in Europe [9], this amounts to a prevalence of 1-9/100,000 population. The 
epidemiological study by Boldingh et al. [10] found a prevalence of between 11.1 and 14.3 per 100,000 for AChR-positive myasthenia gravis cases. In Canada, there was a small increase in prevalence from $16.3 / 100,000$ (1996) to $26.3 / 100,000$ (2013) which is somewhat related to improved treatment options and diagnostics [11]. On the other hand, new cases of myasthenia gravis between 2.3 and 2.7/100,000 annually seem to be more stable [11].

\section{Approved substances and those included by the Joint Federal Committee (G-BA) and in the Medicinal Products Directive (AM-RL)}

In German some substances have been approved by the Federal Institute for Drugs and Medical Devices (BfArM) for the application area myasthenia gravis ( $>$ Fig. $\mathbf{1}$ ). These are generally legacy substances. However, these were only partially dealt with in the Preparations committees (in this case Preparation Committee B3 of the Federal Health Office), the work of which was discontinued in 1986 [12]. In 2004 azathioprine was approved in the post-authorization procedure for myasthenia gravis. No subsequent approval was requested for ambenonium chloride which was fictively approved in Germany until the end of 1991. Although it is possible to use ambenonium chloride in Germany, an import (according to Drug Law Section 73 para. 3) from one of the EU member states (France, Poland, Hungary) is required to treat the use as an off-label application.

Since the introduction of the "Off-label" expert groups at the BfArM in 2006, regulation of some substances by decisions of the Joint Federal Committee according to Section 35 c, V of the Social Security Code could be found [13] for mycophenolate mofetil (MMF, 12/03/2013) and intravenous immunoglobulin G (IVIg, 9/10/2013). According to AM-RL Annex VI Part A, these may be used for off-label applications without prior request by the attending physician. Thus, the basic therapy was extended so that it could be described as "optimized standard therapy" (see $>$ Fig. 1). The costs are assumed by the GKV and do not lead to recourse [4].

\section{Practical application}

The substances approved in Germany for myasthenia gravis are treated as basic therapy in the current guidelines of the German Neurological Society (DGN) (AWMF registry number: 030/087) [14]. Not all approved substances are actually used to treat myasthenia gravis.

A Norwegian study [15] analyzed the entire spectrum of prescribed drugs among 850 patients. Of 527 myasthenia gravis patients receiving immunosuppressants (IS), the ratio of azathioprine to steroids was 32:60\%; a survey in Germany revealed a ratio of 77:14\% [16] (see - Table 1). A survey of the German Myasthenia Society (DMG) regarding long-term therapy exhibited a distribution ratio of drugs as show in > Fig. 2.

Detection of new antibodies in cases of myasthenia gravis resulted in identification of new variants (e. g., MuSK-positive MG [17]) which require a significantly different dosage and therapy planning compared to AChR-antibody-positive myasthenia $[18,19]$.

\section{Regulation Concept for Off-label Use}

Rare disease (orphan) designation

The prerequisites for improving the evidence for new substance approval for a rare disease have previously been elaborate, presum- ably not very attractive to a pharmaceutical manufacturer to undertake classical random clinical trials, e. g., to expand the current authorization. In its “Rapid Report”, the German Institute for Quality and Efficiency in Healthcare (IQWiG) indicated that the methodological procedure regarding rare diseases is practically the same as for non-rare diseases [3]. However, even with the concept of a rare disease there are "singular cases" [10] or "individual cases", which are considered "ultra-rare diseases" (prevalence: $<1: 10,000$ ), including, for example MuSK-positive myasthenia gravis (prevalence $0.05-0.65 / 100,000$ [10]) or congenital myasthenic syndrome (CMS) [20]. Consequently, Vrinten et al. [21] introduced the study concept of a so-called single-case trial, using ephedrine as an example of add-on therapy for MG. For medicinal products, the concept of "rare disease (orphan) designation" (formerly: orphan drug designation) was introduced by the EMA for a rare disease and its related constellations. The effect of this, for example, was that 3,4-diaminopyridine, which has been known and used for over 40 years and which according to the German New Prescription Formulary (NRF, no. 22.3) can be prepared by a pharmacy; it can also be used to treat Lambert-Eaton myasthenic syndrome (LEMS) as a guideline-compliant therapy and has been approved by the EMA as an orphan drug (amifampridine). The high costs of this procedure as well as the procedure itself has been critically discussed [22].

Among the monoclonal antibodies currently in clinical trials, eculizumab has received the rare disease (orphan) designation for myasthenia gravis (EMA: 7/29/2014); this means that the manufacturer has provided proof to the EMA that it provided a "significant benefit" (Par. 3 of EU directive 141/2000) [23, 24]. In the meantime, eculizumab has been approved for the treatment of "therapy-refractory generalized MG with proven antibodies against acetylcholine receptor protein" (8/14/2017) [25]. Following approval by the EMA as an orphan drug in Germany, the procedure according to the Pharmaceuticals Market Reorganisation Act (AMNOG) for assessing an added benefit is only required if sales of the drug exceed a specified amount (currently EUR 50 million). As a legacy drug, eculizumab is not subject to an AMNOG procedure.

Function of the neurology/psychiatry expert group at the federal institute for drugs and medical devices (BfArM)

In Germany, regulation of off-label use is following the approach in accordance with Social Security Code V. The Joint Federal Committee (G-BA) assign the Off-label Neurology / Psychiatry Expert Groups, attached to the BfArM, founded in 2006 to evaluate substances used off-label. The assigment requires a request to the Joint Federal Commission (G-BA) made by the German health insurance $(\mathrm{GKV})$, Federal medical association (KBV) or self-help groups to review the prerequisites for the procedure. The assessment is prepared by one of the external experts nominated by the relevant expert group. The subsequent evaluation by the expert group is then prepared according to a coordinated method paper and sent to the G-BA as a consolidated evaluation proposal, which then passes a resolution following an decision-making procedure. Either the substance is thus included in Part A of AM-RL Annex VI (positive) or in Part B (no off-label application possible). Substances in Part A can be prescribed directly without prior request by the GKV and costs assumed by the GKV, if the guidelines specified in the G-BA decision are adhered to, which are more or less an extension of the tech- 


\begin{tabular}{|c|c|c|c|c|}
\hline Off-label & On-label & Orphan drug & Guideline & Measure \\
\hline & & & & Thymectomy \\
\hline & & & & Steroids \\
\hline & & & & Azathioprine \\
\hline GKV & & & & Methotrexate \\
\hline AM-RL + & & & & Mycophenolate mofetil ${ }^{* * *}$ \\
\hline GKV & Japan & & & Tacrolimus \\
\hline GKV & & & & Cyclosporin A \\
\hline GKV & & & & Cyclophosphamide \\
\hline AM-RL + & & & & High-dose IVIg (** $^{* *}$ \\
\hline \multirow[t]{2}{*}{ GKV } & & & & Intermittent IVlg \\
\hline & & & & Immunoadsorption IAD \\
\hline \multirow[t]{2}{*}{ GKV } & & & & Intermittent IAD \\
\hline & & & & Plasma replacement \\
\hline \multirow[t]{2}{*}{ GKV } & & & & Rituximab \\
\hline & & EMA & & Eculizumab \\
\hline \multirow[t]{5}{*}{ GKV } & & & & Other monoclonal antibodies \\
\hline & & & & Anti-sense therapy \\
\hline & & & & Etanercept \\
\hline & & & & Therapeutic vaccination \\
\hline & & & & Pyridostigmine bromide \\
\hline \multirow[t]{3}{*}{ GKV } & France & & & Ambenomium chloride* \\
\hline & & & & Edrophonium chloride ${ }^{* *}$ \\
\hline & $\begin{array}{l}\text { New Prescription } \\
\text { Formulary No. } 22.3\end{array}$ & EMA & & 3,4-Diaminopyridine (DAP) \\
\hline
\end{tabular}

- Fig. 1 Overview and approval status of treatment procedures for myasthenia gravis (pharmacotherapy, interventions and surgeries) following the guideline (GL) of the German Neurological Society (gray column). Orphan drug status and on-label use are indicated by region (green). Substances in on-label use and off-label use as approved substance according to the Medicinal Products Directive (AM-RL +$)$ are shown in boldface. Other off-label use requires application to the German health insurances (GKV), possibly legal recourse in case of non-approval by the statutory health insurance. * only approved for myasthenia gravis in France, Poland, Hungary; ${ }^{* *}$ only approved in Spain for a pharmacological test; ${ }^{* * *}$ by decision of the Joint Federal Committee (G-BA) included in AM-RL Annex VI Part A, reimbursable and prescribable in off-label use without application.

nical information. Treatment with MMF and IVIg that can be ordered and costs assumed by the GKV is a positive result of this procedure, but requires special instruction and monitoring $([3,4]$;

- Table 2).
If in an individual case, a preliminary qualified application must be made to the relevant health insurance if the clinical progression requires expansion of the treatment with off-label use of substances, as indicated with second-line substances according to the cur- 
- Table 1 Use of corticoids and immunosuppressants for the treatment of myasthenia gravis. Norway in the period 2004-2010: of 830 MG patients on pyridostigmine, 636 use an immunosuppressant (data from [15]). In Germany, 744 of 899 MG patients interviewed in 2007 took immunosuppressants (unpublished data).

\begin{tabular}{|c|c|c|c|c|}
\hline \multirow[b]{2}{*}{ Immunosuppressives } & \multicolumn{2}{|c|}{$\begin{array}{l}\text { Norway } \\
2004-2010\end{array}$} & \multicolumn{2}{|c|}{ Germany 2007} \\
\hline & $N=636$ & $\%$ & $N=744$ & $\%$ \\
\hline Azathioprine & 201 & 32 & 570 & 77 \\
\hline Corticosteroid & 387 & 61 & 107 & 14 \\
\hline Mycophenolate mofetil & 23 & 4 & 42 & 6 \\
\hline Cyclosporine & 15 & 2 & 15 & 2 \\
\hline Methotrexate & 10 & 2 & 8 & 1 \\
\hline Cyclophosphamide & 0 & 0 & 2 & $<1$ \\
\hline
\end{tabular}

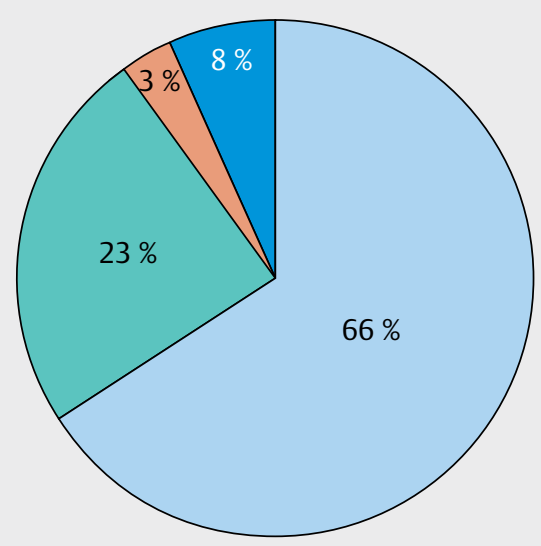

Steroid plus pyridostigmine

Steroid plus azathioprine plus pyridostigmine

Azathioprine plus pyridostigmine
MTX, cyclosporin A, cyclophosphamide plus pyridostigmine

- Fig. 2 Spectrum and proportion of drugs used in long-term therapy in 763 myasthenic patients. German Myasthenia Society (DMG) modified according to data taken from Wöhrle [16].

- Table 2 Recommendations for the indication and monitoring of a planned treatment [3].

1. Comprehensive documentation of therapy resistance

2. Comprehensive information

3. Safety advisory

4. Information for decision-making

5. Written informed consent

6. Comprehensive documentation of progression (exact, closely monitored, personal)

rent guideline, but which so far have not been evaluated by the expert group. The application must provide scientific evidence, e.g., according to the current guideline as well as the state of clinical expertise with respect to an case-specific analysis $[1,13,14]$. The application is then reviewed by the MDK and generally approved if the criteria of the Social Security Code opinion dated 3/19/2002 have been met (so-called Sandoglobin opinion) or the criteria of the Nikolaus opinion (12/6/2006) have been fulfilled [4-6]. Unfortunately, the experience of the treating physician and/or myasthenia experts is often not sufficiently taken into consideration. A negative decision can mean that - in urgent cases - an injunction has to be pursued.

In the long term, according to the EU guidelines, the establishment of disease-specific "Centers of Expertise" (COE) is planned for such tasks as initiated in Germany by the "National Alliance for People with Rare Diseases" (NAMSE). Currently queries should be addressed to the certified integrated myasthenia center (IMZ; [26]) or specialized myasthenia outpatient clinics.

\section{Myasthenia Gravis}

\section{Optimized standard therapy}

Optimized standard therapy is an extended basic therapy, which in addition to the symptomatic, on-demand administration of pyridostigmine bromide [27] usually includes anti-inflammatory treatment with steroids (therapy goal: induction of remission), accompanied by additive long-term immunosuppression with azathioprine, which has a steroid-sparing effect and is intended to maintain and improve the achieved remission (therapy goal: remission maintenance). Azathioprine can be replaced by mycophenolate mofetil, in the case of azathioprine intolerance or ineffectiveness with appropriate dosage and duration of use (or even in case of unfeasible reduction of steroids below the Cushing threshold). In the event of myasthenic exacerbation IVIg can be used as an outpatient add-on therapy for stabilization $[28,29]$.

\section{Non-responders}

About $14.8 \%$ of all myasthenia gravis patients cannot sufficiently benefit from optimized standard therapy $[14,30,31]$. These patients are frequently steroid responders, that is, they improve and are stable with steroid dosage which generally lies above the Cushing threshold, or they require IVIg or plasma replacement interventions. Nevertheless, they should be regarded as non-responders under optimized standard therapy, since the feared adverse drug reactions of a usually required long-term therapy with steroids must not be accepted [14, 28, 32-34]. In such cases, immunosuppressive therapy with off-label use is employed (second line, e. g., methotrexate, cyclosporin A, tacrolimus) (see $>$ Table 1). This therapy corresponds to an individual treatment, which requires comprehensive clarification and documentation ( $\vee$ Table $2 ;[3,4,14])$. The situation with respect to juvenile myasthenia gravis is rather more complex [35].

\section{Therapy-refractive myasthenia gravis}

There are a few patients, probably well below $15 \%$ with generalized MG, who do not sufficiently improve by expanded optimized standard therapy, that is, even after the use of second-line immunosuppressants, such as methotrexate or cyclosporin A. This constellation occurring in the course of generalized, often fluctuating gen- 
- Table 3 Criteria for therapy-refractory myasthenia gravis ([30,31].

1. Severe, generalized MG with insufficient response to adequate dosage and duration of immunosuppressive drug

2. Frequent myasthenic crises and/or sever exacerbation

3. Repeated interventions (IVIg or IAD or plasma replacement)

4. Risk of intermittent home ventilation

5. Severe adverse drug reactions under optimized standard therapy

6. Increased individual risks (e. g., comorbidities)

7. Contraindications for guideline-based treatment

eralized MG is referred to as therapy-refractory myasthenia gravis. There is no consensus regarding individual criteria for therapy-refractory generalized myasthenia gravis (TRgMG) (see > Table 3 $[30,31,34,39]$. Therefore, when reviewing the evidence and weighing the benefit/risk profile prior to an intended therapeutic step, the individual case (e.g., MuSK-positive myasthenia gravis) must be established. Due to the varying individual distribution of muscle weakness and crises, this is useful when treating MG. An individual risk/benefit assessment is an additional very important decision criterion, not least for the participative patient decision [14, 19, 28, 32].

Expanded escalation therapy may then mean that in addition to immunosuppressive therapy, there is either indication for a stabilizing long-term therapy by intermittent IVIg administration, or more rarely, consideration should be given to long-term IAD [36] or long-term plasma replacement as an add-on therapy. Likewise, an attempt at remission induction by using monoclonal antibodies, e. g., rituximab in off-label use has shown its value [14, 17, 19 , 37-39]. For over 10 years, the use of rituximab, especially for MuSK-positive myasthenia gravis has been a favored successful strategy [39]. Its effectiveness and risk/benefit assessment has been largely seen positively. Of the other monoclonal antibodies mentioned in the guidelines, eculizumab is approved by the EMA and acts as an add-on therapy for ongoing treatment of TRgMG with demonstrated AChR antibodies. As a legacy substance eculizumab is not subject to the AMNOG procedure. The diversified indication within German treatment practice, which is based on an expertise of well over 10 years ("well-established use"), has just begun. Therefore, many questions are still unanswerable, the application will be limited to special cases with generalized MG.

There are very rare constellations of definitely insufficiently treatable myasthenia gravis, the development of which is not always understood (so-called burned-out myasthenia, areactive MG). It remains to be seen whether such disease progressions will be avoided in the future with current therapeutic possibilities. Respiratory paralysis is the most severe impairment. Indication for home ventilation in such cases must be determined in conjunction with neurologists and pulmonologists [41].

\section{Lambert-Eaton Myasthenic Syndrome (LEMS)}

Immunosuppressive treatment of idiopathic and paraneoplastic LEMS (prevalence: 0.1-0.9/100,000 [9]) with steroids and/or azathioprine is used analogously to myasthenia gravis patients $[29,42]$, although within the framework of off-label use, since there is no explicit approval for this area of application due to the absence of study data. This therapy is within guidelines, however. Symptomatic therapy uses 3,4-diaminopyridine (amifampridine), which is available as a formulation according to the New Prescription Formulary (NRF $22.35 \mathrm{mg}$ or $10 \mathrm{mg}$ ) or as a single-source drug. The EMA issued approval in 2010. In the UK, for example, pricing has led to the previous framework rising from approx. $£ 600$ per year per patient to $£ 60,000$; the situation seems the same in Germany as well as recently in the United States [22].

\section{Congenital Myasthenic Syndromes (CMS)}

Congenital myasthenic syndromes are genetically determined together with other ultra-rare diseases (prevalence: 1-2/500,000 [9]), with an increasingly widening spectrum [20]. Apart from approved therapy with pyridostigmine bromide, there are no additional drugs available that are explicitly approved for one of these CMS diseases. However, pyridostigmine bromide is useful for only a few congenital myasthenic syndromes. Administration of 3,4-diaminopyridine (amifampridine), which is effective in some forms of CMS due to the well-known pathophysiology, must be prescribed as an off-label use upon application to the health insurance. Other substances, some of which can only be obtained through the international pharmacy (for example ephedrine $[32,40,43]$ ) pose the same requirements for doctors and patients as off-label use. This kind of sustained therapy after a differentiated examination, such as in a neuropediatric medical center or a suitable specialized outpatient muscle clinic, is required in any case [44].

Pragmatic approach to the use of off-label substances for treating myasthenia Gravis, LEMS and CMS

1. The immunosuppressants methorexate and cyclosporin A are always used off-label to treat myasthenia gravis or LEMS, except in cases of rheumatic secondary disease. There are only exceptionally problems with reimbursement, since these are recognized drugs for treating rheumatism with widespread use, although in this case are used off-label, there is familiarity and understanding on the part of the MDK.

2. Intermittent therapy with IVIg in the long term is a rare indication as add-on therapy in disease phases where stabilizing intervention is required. Since this is so far an off-label use, the individual case has to be differentiated, and rejection by the MDK should unfortunately be expected.

3. The off-label use of rituximab is an attempt to induce remission in the case of therapy-refractive myasthenia gravis. This significant escalation in the ultra-rare disease area should be applied for in advance of long-term outpatient therapy. Here the reputation of a specialized center is recommended.

4. The indication for eculizumab as an add-on therapy in refractory generalized myasthenia gravis is limited to patients with verified acetylcholine receptor-antibodies.

5. The indication for an add-on therapy with eculizumab should be made via designated myasthenia centers.

6. 3,4-diaminopyridine (amifampridine) can still be produced to treat LEMS by pharmacies according the New Prescription Formulary (NRF no. 22.3). The outpatient prescription of the original preparation requires a corresponding procedure to obtain a special status of the practice (so-called cost-intensive patient). 


\section{Conflict of Interest}

The author is a member of the Transparency and Independence commission of the Drug Commission of the German Medical Association and member of the Off-label Expert Group (Neurology/ psychiatry) of the Federal Institute for Drugs and Medical Devices.

\section{References}

[1] Bundesinstitut für Arzneimittel und Medizinprodukte (BfArM) www. bfarm.de

[2] Erhebung des Off-Label-Use ind der EU (2017) www.https://ec. europa.eu/...2017_2_28_finalstudy_report_on_off-label_use.pdf

[3] Janzen RWC, Ludwig WD. Off-Label-Therapie: Aktuelle Probleme aus Sicht der Arzneimittelkommission der deutschen Ärzteschaft. Z Rheumatol 2012; 71: 108-118

[4] Clemens T. Zulässigkeit von Arzneiverordnungen und Kostenregresse gegen Ärzte - Off-Label-Use und Unlicensed Use. GesR 7/2011 397-409

[5] Bundessozialgericht ( B 1 KR 1/16R) vom 13.12.2016

[6] Bundesverfassungsgericht (1 BvR 452/17) vom 11.4.2017

[7] Janzen RWC. Off-Label-Use im Behandlungsalltag. Arzneiverordnungen in der Praxis 43: 127-132

[8] European Federation of Neurological Associations (EFNA) board Declaration: Good Off-Label Use Practices (GOLUP) 2017 www. braincouncil.eu/activities/golup

[9] Orphanet ( www.orpha.net/national/DE-DE/index/startseite )

[10] Boldingh M, Maniaal AH. Bruborg et al. Geographical distribution of myasthenia gravis in Northern Europe-Results from a population-based study from two countries. Neuroepidemiology 2015; 44: 221-231

[11] Breiner A, Widdifield J, Katzberg HD et al. Epidemiology of myasthenia gravis in Ontario, Canada. Neuromuscul Dis 2016; 26: 41-46

[12] Müller-Oerlinghausen B, Schulz M. Abruptes Ende der systematischen Aufbereitung des Altarzneimittelmarkts im Bereich Psychiatrie, Neurologie und Anästhesie. Nervenarzt 1996; 67: 1040 - 1045

[13] Gemeinsamer Bundesausschuss (G-BA) (www.g-ba.de )

[14] Leitlinien der Deutschen Gesellschaft für Neurologie (DGN), 1.4.2017 ( www.dgn.org )

[15] Andersen JB, Owe JF, Engeland A et al. Total drug treatment and comorbidity in myasthenia gravis: A population-based cohort study. Eur ] Neurol 2014; 21: 948-955

[16] Wöhrle G. Untersuchungen zu Epidemiologie und Verlauf der Myasthenia gravis in Deutschland. Dissertation, TU Darmstadt 2000

[17] Iorio R, Damato V, Albioni PE et al. Efficacy and safety of rituximab for myasthenia gravis: a systematic reviews and meta-analysis. J Neurol 2015; 262: 1115-1119

[18] Gilhus NE, Verschuur I]. Myasthenia gravis: Subgroup classification and therapeutic strategies. Lancet Neurol 2015; 14: 1023-1036

[19] Dalakas MC. Novel future therapeutic options in myasthenia gravis. Autoimmun Rev 2013; 12: 936-941

[20] Engel AG, Shen XM, Selcen D et al. Congenital myasthenic syndromes: Pathogenesis, diagnosis, and treatment. Lancet Neurol 2015; 14: 420-434

[21] Vrinten C, Lipka AF, van Zwet EW et al. Ephedrine as add-on therapy for patients with myasthenia gravis: Protocol for a series of randomised, placebo-controlled n-of-1 trials. BMJ Open 2015; 5: e007863

[22] Burns TM, Smith GA, Allen JA et al. Editorial by concerned physicians: Unintended effect of the Orphan Drug Act on the potential cost of 3,4-diaminopyridine. Muscle Nerve 2016; 53: 165-168
[23] Howard JF, Barohn RJ, Cutter GR et al. A randomized, double-blind, placebo-controled phase II study of eculizumab in patients with refractory generalized myasthenia gravis. Muscle Nerve 2013; 48: 76-84

[24] Howard JF Jr, Freimer M, O'brien F et al. QMG and MG-ADL correlations: Study of eculizumab treatment of myasthenia gravis. Muscle Nerve. 2016; 56: 328-330

[25] www.ema.eu/docs/en_GB/document_library/summary_of_opinion/000791/WC500229915.pdf (30.07.2017)

[26] Bungard S, Rohn H, Döbler K. Zertifizierung von Myastheniezentren: Entwicklung und Implementierung eines Zertifizierungsprozesses für Selbsthilfeorganisationen. Z Evid Fortbild Qual Gesundhwes 2011; 105: 49-53

[27] Pohanka M. Acetylcholinesterase inhibitors: A patent review (2008-present). Exp Opin Ther Pat 2012; 22: 871-886

[28] Henze T, Janzen RWC, Schumm F et al. Immuntherapie bei Myasthenia gravis und Lambert-Eaton-Syndrom. Teil 1: Medikamentöse Immunsuppression. Akt Neurologie 2010; 37: 505-517

[29] Urban P, Jacobi C, Jander S. Myasthenia gravis: Treatment standards and patient-tailored therapy. Akt Neurol 2018; 45: 253-262

[30] Silvestri N], Palace JA. Recognizing refractory myasthenia gravis. Fast Facts. Health Press Limited; Oxford: 2017

[31] Suh J, Goldstein JM, Nowak RJ. Clinical characteristics of refractory. myasthenia gravis patients. Yale J Biol Med 2013; 86: 255-260

[32] Sieb JP. Myasthenia gravis: An update for the clinician. Clin Exp Immunol 2014; 175: 408-418

[33] Alshekhlee A, Miles JD, Katirji B et al. Incidence and mortality rates of myasthenia gravis and myasthenic crisis in US hospitals. Neurology 2009; 72: $1548-1554$

[34] Silvestri N], Wolfe GI. Treatment-refractory myasthenia gravis. J Clin Neuromuscul Dis 2014; 15: 167-178

[35] Della Marina A, Tripp H, Lutz S et al. Juvenile myasthenia gravis: recommendations for diagnostic approaches and treatment. Neuropediatrics 2014; 45: 75-83

[36] Wagner S, Janzen RWC, Mohs C et al. Langzeitbehandlung der therapierefraktären Myasthenia gravis mittels Immunadsorption. Dtsch Med Wochenschr 2008: 133: 2377-2382

[37] Robeson KR, Kumar A, Keung B et al. Durability of the rituximab response in acetylcholine receptor autoantibody-positive myasthenia gravis. JAMA Neurol 2017; 74: 60-66

[38] Tandan R, Hehir MK, Waheed W et al. Rituximab treatment of myasthenia gravis: A systematic review. Muscle Nerve 2017; 56: 185-196

[39] Hoffmann S, Meisel A. Escalation strategies in the treatment of refractory myasthenia gravis. Akt Neurol 2018; 45: 294-297

[40] Rodríguez Cruz PM, Palace J, Ramjattan H et al. Salbutamol and ephedrine in the treatment of severe AChR deficiency syndromes. Neurology 2015; 85: 1043-1047

[41] Fernandes Oliveira E, Nacif AH et al. Sleep disorders in patients with myasthenia gravis: a systematic review. J Phys Ther Sci 2015; 27 : 2013-2018

[42] Kohler S, Meisel A. The Lambert-Eaton myasthenic syndrome: An overview. Akt Neurol 2018; 45: 298-304

[43] Vrinten C, van der Zwaag AM, Weinreich SS et al. Ephedrine for myasthenia gravis, neonatal myasthenia and the congenital myasthenic syndromes. Cochrane Database Syst Rev 2014; 12: CD010028

[44] Schara U, Della Marina A, Abicht A. Congential myasthenic syndromes: current diagnostic and therapeutic approaches. Neuropediatrics 2012; 43: 184-193 